\title{
Evaluación de estrategias formativas para mejorar las actitudes hacia las matemáticas en secundaria
}

\section{Evaluation of training strategies to improve attitudes towards mathematics in secondary}

\section{Rosa María González Jiménez ${ }^{1}$}

Resumen: Diseñamos, implementamos y evaluamos (proceso y resultados) un programa de formación Actitudes hacia las Matemáticas con perspectiva de Género (PAMG) dirigido a estudiantes de doce escuelas secundarias públicas ubicadas en la Ciudad de México (ocho de ellas vespertinas y con bajas calificaciones en la prueba ENLACE), cuyo propósito fue probar estrategias didácticas para mejorar las actitudes del alumnado hacia las matemáticas, desde una perspectiva de género. El programa consta de cinco estrategias (persuasión, metacognición, conflict cognitive e historia de vida) en nueve actividades didácticas con seis horas de duración. Para evaluarlo construimos una metodología mixta que incluye un análisis interpretativo del proceso (notas y videograbación de las sesiones) y un diseño cuasiexperimental con análisis estadístico (resultados). En lo general, el PAMG cambió positivamente las actitudes de estudiantes en la mitad de las escuelas, según el análisis de algunos factores que incidieron en aquellos grupos que no experimentaron cambios significativos. Cualitativamente identificamos que hablar en primera persona acerca de las matemáticas y buenas relaciones socio-afectivas en el grupo, inciden positivamente en el trabajo en el aula.

Fecha de recepción: 21 de octubre de 2017. Fecha de aceptación: 10 de septiembre de 2018.

1 Universidad Pedagógica Nacional, México. rosamaria@upn.mx orcid.org/0000-0003-1894-1282 
Palabras clave: actitudes; matemáticas; estudios de género; secundaria; intervención.

\begin{abstract}
We design, implement and evaluate (process and results) a training program (pamg) for students of twelve public high schools located in the City of Mexico (eight of them evening and low results in the ENLACE test), whose purpose was to improve and the attitudes of students towards mathematics from a gender perspective. The program consists of five strategies (persuasion, metacognition, conflict cognitive and life history) in nine didactic activities with six hours duration. To evaluate the pamg we build a mixed methodology that includes an interpretive analysis of the process (notes and videotaping of the sessions) and a quasi-experimental design with statistical analysis (results), identifying those specific strategies that positively improved attitudes girls and boys. Qualitatively, we identify that speaking in the first person about mathematics and good social-affective relationships in the group, positively impact on the work in the classroom.
\end{abstract}

Keywords: attitudes; mathematics; gender studies; junior high school; intervention.

\title{
INTRODUCCIÓN
}

Es conocida la baja proporción de estudiantes que ingresan al campo de las matemáticas, en comparación con otras disciplinas (Menchaca, 2000; OCDE, 2016); particularmente, se sabe de la subrepresentación de mujeres matemáticas en estudios superiores (González, 2006; Espinosa, 2010). Como parte de las políticas de género del Instituto Nacional de las Mujeres (Inmujeres), nos invitaron, ${ }^{2}$ como expertas, a realizar una intervención cuyo objetivo era mejorar las actitudes de las alumnas de secundaria hacia las matemáticas. El trabajo implicó tres planos articulados: a) diseño del programa; b) implementación en doce escuelas secundarias, y c) evaluación (proceso y resultados), que presentamos en este reporte.

2 Hace veinte años iniciamos una Especialización en Estudios de Género en Educación en la Universidad Pedagógica Nacional, una de cuyas líneas de investigación es precisamente Género y matemáticas. 
Esta intervención la consideramos un ejercicio sistemático y riguroso que nos permitió -además de probar en el campo algunas estrategias educativas que trabajamos en la formación de especialistas en estudios de género en educación desde la intersubjetividad y la dimensión afectiva (González, 2002; 2012)- comprender mejor los procesos que inciden en la formación de actitudes hacia las matemáticas. Por lo demás, el programa es un ejemplo, entre otros, que el profesorado puede diseñar cotidianamente en su salón de clase teniendo en cuenta las características de sus estudiantes y el contexto de su escuela, retomando algunas de las estrategias didácticas que probamos y dando rienda suelta a su creatividad.

El aprendizaje de las matemáticas se consideró, por mucho tiempo, como un problema sólo cognitivo. Pero hace más de dos décadas, McLeod (1992), una autoridad en educación matemática, empezó a enfatizar la importancia de los afectos, lo que incrementó el interés de los especialistas por el llamado dominio afectivo de la enseñanza, referido a un conjunto de aspectos entre los que se incluyen actitudes, creencias y emociones, constructos que, si bien tocan todos los campos de conocimiento, en educación básica repercuten particularmente en el aprendizaje de las matemáticas (Leder y Forgasz, 2002; Ursini, Ramírez, Rodríguez, Trigueros y Lozano, 2010, y Ursini, Montes, Ramírez y García, 2012).

Hay diferentes perspectivas teóricas acerca de la dimensión emocional; ${ }^{3}$ según la nuestra, la dimensión afectiva se fundamenta en el psicoanálisis freudiano ${ }^{4}$ (como teoría de la intersubjetividad y la representación-afecto), del cual se deriva una estrategia didáctica específica para trabajar esta dimensión.

\section{DISEÑO Y EVALUACIÓN DE ESTRATEGIAS ACTITUDINALES}

Hay dos concepciones encontradas acerca de cómo programar y evaluar estrategias formativas. Por una parte, dicha actividad se concibe como la aplicación de principios susceptibles de ser explicados causalmente, que se interesa por los resultados y utiliza diseños cuasiexperimentales para medir si hubo cambios y en qué sentido (Murillo, 2008). Por otra parte, se entiende como una tarea

\footnotetext{
${ }^{3}$ Véase la revisión de McLeod (2002) y Gil, Blanco y Guerrero (2005).

${ }^{4}$ A diferencia de otros enfoques que separan lo cognitivo de lo afectivo, para el psicoanálisis la representación (ideas, imágenes o fantasías) está unida al afecto; este último, con una dimensión cuantitativa -más o menos intensa- y otra cualitativa que se manifiesta como placer o displacer (Chiozza, 1998). Cuando la racionalidad falla, surge el afecto.
} 
artística y creativa, imposible de definir en términos de causa y efecto, cuyo interés se centra sobre todo en el sentido, las relaciones y el proceso desde un contexto determinado, valiéndose de un enfoque interpretativo (Arthur, Waring, Coe y Hedges, 2012).

Efectivamente, toda puesta en acto de un programa educativo es singular (en ocasiones, creativa); sin embargo, el diseño de un programa formativo implica algunas regularidades (los objetivos, las estrategias didácticas, la experiencia profesional del docente) y varias situaciones contextuales cambiantes (estudiantes con diferentes historias de vida, grupos escolares con distintas relaciones y trayectorias académicas, condiciones materiales del aula, actitudes hacia el aprendizaje, etc.). El profesional de la educación, en diversos contextos y grupos escolares, sabe cómo proceder para que un programa cumpla con los propósitos de formación.

El Inmujeres se interesaba especialmente en un diseño experimental que llegara a la mayor cantidad de estudiantes; a nosotras nos interesaba sobre todo comprender qué ocurría en el proceso, por lo que vinculamos metodológicamente un diseño cuasiexperimental con análisis estadístico (resultados) como un análisis interpretativo (del proceso), articulación no exenta de dificultades ${ }^{5}$ que busca acercar posiciones dicotómicas y excluyentes (Bolívar, 2005), respetando la lógica (inducción para el diseño cuasiexperimental y conjetural para la interpretación de los videos considerados textos) de cada procedimiento. Por cuestión de espacio, este reporte se centra en el análisis de resultados.

La investigación implicó tres planos reflexivos-programáticos articulados: 1) diseño del Programa de Actitudes hacia las Matemáticas con perspectiva de Género (PAMG), 2) implementación del programa en doce escuelas secundarias de la Ciudad de México y 3) diseño metodológico de la evaluación (proceso y resultados) del PAMG, que a continuación se describen.

\section{DISEÑO DEL PROGRAMA}

a) Construcción lógico-conceptual del modelo "Cambio de actitudes hacia las matemáticas con perspectiva de género".

\footnotetext{
${ }^{5}$ No ignoramos los principios epistémicos y ontológicos diferentes de una y otra posición.
} 
b) Con base en el modelo anterior, diseño del programa "Actitudes hacia las matemáticas con perspectiva de género" (PAMG), dirigido a estudiantes de $1^{\text {음 }}$ de secundaria.

\subsection{FUNDAMENTACIÓN DEL PAMG}

El diseño de cualquier programa parte del supuesto de que la formación puede incidir en la transformación del alumnado al que se dirige; el cambio lo entendemos en sentido metafórico como "mirar desde otra posición"; esto es, resignificar $^{6}$ (las matemáticas, en este caso) y resignificar-se en relación con este saber-hacer. Este segundo aspecto es el que abordamos. Además de revisar la literatura, analizamos semánticamente el concepto de actitud, lo que nos permitió formular algunos supuestos previos acerca de cómo orientar el cambio que se pretende. Enseguida se presenta una síntesis de algunos fundamentos teóricos del PAMG.

\section{CAMBIO DE ACTITUDES HACIA LAS MATEMÁTICAS}

El término actitud se usa para describir la respuesta evaluativa (positiva, negativa, indiferente) de alguien hacia una situación, persona u objeto (Bolívar, 2000). Las actitudes hacia las matemáticas son un constructo multidimensional que incluye convencionalmente, en el caso de estudiantes, sus creencias acerca de las matemáticas como campo de conocimiento (nivel de dificultad y valor o utilidad) o actividad escolar (interesantes, aburridas), los afectos negativos que provoca en algunos de ellos resolver problemas matemáticos (ansiedad), los afectos generados por sus experiencias previas (gusto, disgusto), las convicciones que tienen acerca de su propia capacidad para las matemáticas (autoconcepto) y su tendencia a considerarlas como una actividad intelectual y profesional propia para hombres (estereotipos de género) (Eccles-Parson, Kaczala, Goff y Futterman 1982; González, 2004a).

${ }^{6}$ Trabajamos también con las y los docentes de matemáticas de las secundarias seleccionadas desde la práctica reflexiva (saber desde la acción) de Donald Shön (1983); por cuestión de espacio, no se incluye. 
McLeod y McLeod (2002) destacan la confusión conceptual que hay entre actitudes, creencias y afectos, desarrollando un esquema para clasificarlos (figura 1).

Figura 1. Clasificación de conceptos de dominio afectivo

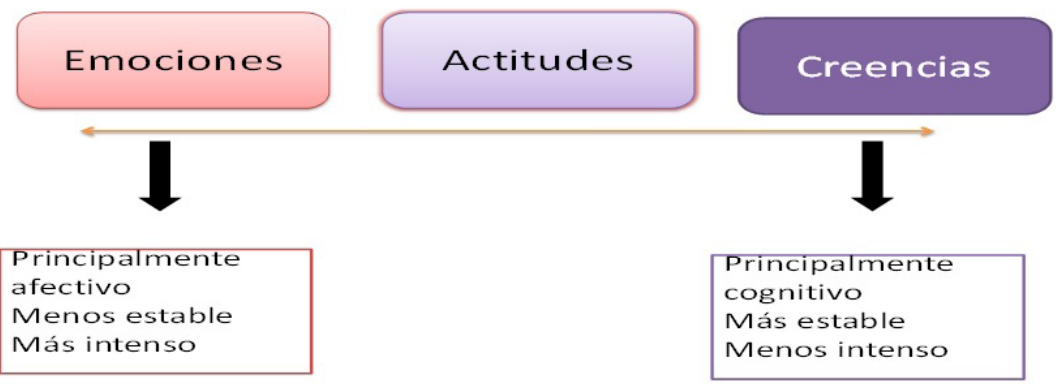

Tanto la información (familia, medios de comunicación) como las experiencias escolares y extraescolares que cada estudiante tiene con las matemáticas van conformando sus creencias respecto a este campo de conocimiento. Sus prácticas en la escuela les generan determinados afectos (gusto, aburrimiento, frustración), los cuales al repetirse llegan a sedimentarse como actitudes que se manifiestan como gusto, rechazo o indiferencia por dicha materia (figura 2). En tanto las actitudes se construyen, son susceptibles de cambio.

Figura 2. Construcción de actitudes

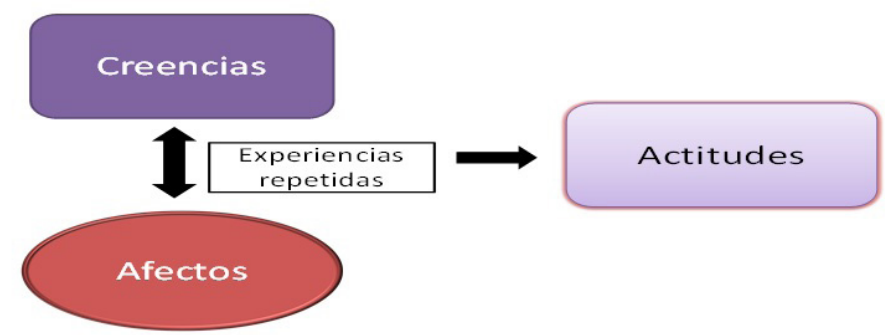

Formulamos la hipótesis de investigación de que si las actitudes hacia las matemáticas en el alumnado se originaron a partir de ciertas experiencias repetidas -que pudieron no ser positivas al generarles creencias y afectos negativos hacia 
la disciplina-, tales actitudes pueden cambiar si se ponen en cuestión sus convicciones negativas y se planean prácticas matemáticas que les resulten interesantes, útiles y lúdicas y que los involucren intersubjetivamente.?

No debatiremos en este espacio la literatura que, durante décadas, ha intentado aclarar si hay diferencias en pruebas de matemáticas entre mujeres y hombres, ya que consideramos que unas y otros no son comparables: partimos de un concepto de ser humano ${ }^{8}$ distante del modelo evolutivo y biológicamente determinado en que se fundamentan las mediciones que comparan personas y comunidades. Por otra parte, dichas diferencias son realmente considerables cuando se valoran condiciones socioeconómicas, carencias en las escuelas, situación étnica o racial (McGraw, Lubienski y Strutchens, 2006) que merecen ser analizadas cuando se traducen en condiciones desfavorables para las mujeres.

La perspectiva de género es una forma de abordar la investigación que cuestiona la organización sexual dicotómicamente estructurada, organización que puede incidir en creencias y actuación en contra de las mujeres, a quienes se les atribuyen habilidades innatas para la vida privada (privilegio del sentimiento que favorece el cuidado de menores, enfermos y ancianos) en contraposición con los hombres, a quienes se les adjudican destrezas para el ámbito público (el gobierno, la razón sobre el sentimiento, la construcción y la fuerza física) (González, 2012) y que castiga para ellos mostrar sentimiento y los responsabiliza del sustento familiar.

En educación hay tres líneas de trabajo en relación con las mujeres y las matemáticas: las alumnas 1) no pueden (cognición); 2) no quieren (motivación), y 3) no les interesan (estereotipos). La primera alude a su capacidad, la segunda a falta de interés y la tercera al ámbito cultural. Con el PAMG buscamos estimular su interés y cuestionar algunos estereotipos: a) que las matemáticas son un campo de conocimiento propio para varones; b) que hasta recientemente las mujeres se iniciaron en este campo de estudio; c) que es un saber abstracto, sin relación con la vida cotidiana.

${ }^{7}$ La intersubjetividad es acontecimiento, no conciencia; se hace consciente y se integra como experiencia en un segundo momento.

${ }^{8}$ Un ser humano no es, está siendo a partir de su origen comunitario, cultura e historia personal (Ricoeur, 2011). Ser hombre o mujer es una pregunta abierta al otro, sin una respuesta definitiva. 


\subsection{Programa Actitudes hacia las matemáticas con perspectiva de Género (PAMG)}

A partir de este modelo diseñamos el PAMG. Decidimos que no enseñaríamos matemáticas, sino que nos centraríamos en las actitudes hacia las matemáticas incorporando la resolución de problemas (por ejemplo, encriptar un mensaje, decidir qué empresa celular les conviene más). Dimos al programa un enfoque constructivista sociocultural y lo situamos en el diálogo, la intersubjetividad y la estética, ${ }^{9}$ delimitando dos competencias: 1) que el alumnado mejorara sus actitudes hacia las matemáticas (valorado a través de un cuestionario); 2) que fueran capaces de reconocer a mujeres matemáticas notables, planteándose los siguientes objetivos:

c) Cuestionar la idea de que las matemáticas son difíciles.

d) Presentar el saber matemático como una actividad cultural lúdica y útil.

e) Cuestionar la tipificación de las matemáticas como un campo de estudio propio para varones.

Además de la presentación, encuadre y evaluación, el PAMG integra nueve actividades programadas con una duración aproximada de seis horas (trabajando dos horas por sesión); varios aspectos se cuidaron en el diseño de cada actividad:

- que el alumnado protagonizara la actividad;

- que se trabajara en equipos y de forma colaborativa;

- que se incluyeran tareas vinculadas con el arte gráfico ${ }^{10}$ y aspectos prácticos;

- que tuviera relación directa con su vida cotidiana;

- que fueran actividades lúdicas y les representaran un reto;

- que propiciaran meta-cognición (reflexión, acerca de sus propios pensamientos).

${ }^{9}$ Lo afectivo, lo sensible ha permanecido prácticamente al margen del discurso educativo, a pesar de su omnipresencia en el aula. La estética, como la recepción sensible de la expresión humana, abre la puerta a una gama de actividades formativas no sólo en torno al arte, sino especialmente en la recepción que el estudiante hace de la actividad que se le propone.

${ }^{10}$ Comprenden la utilidad de la geometría para representar su pensamiento de forma abstracta. 
Se trabajó cada actividad de acuerdo con los objetivos particulares, incorporando diversas estrategias didácticas, algunas dirigidas a las creencias o afectos y una específica para la dimensión intersubjetiva que llamamos historia de vida (figura 3).

Figura 3. Estrategias didácticas para cambio de actitudes

Estrategias didácticas

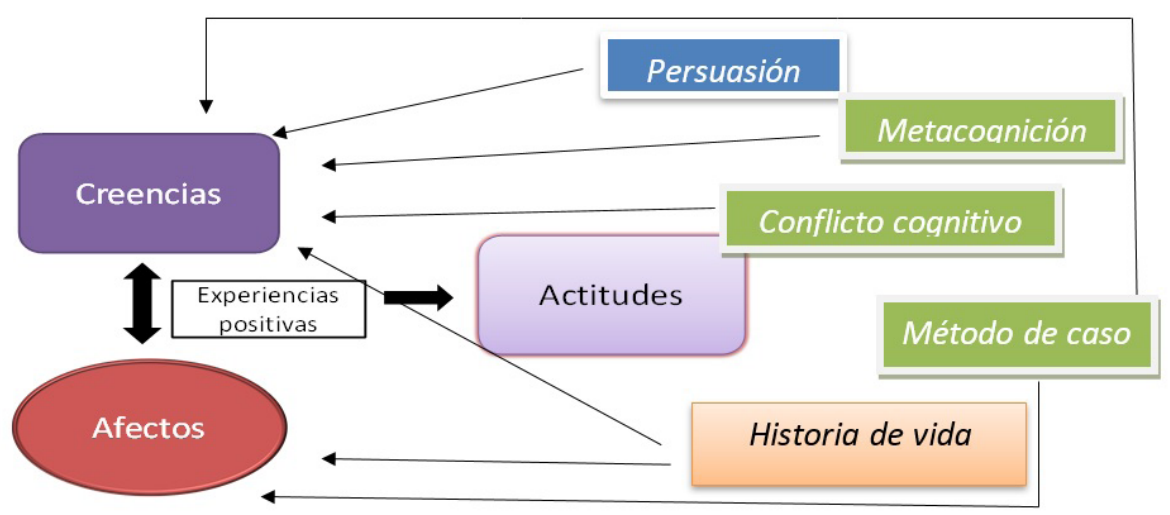

Historia de vida es una estrategia original para mejorar la integración grupal y el cambio de actitudes hacia determinados conocimientos, que ubica en el centro la dimensión intersubjetiva y afectiva ${ }^{11}$ del acto educativo (González, 2012).

El PAMG también incluye tres videos con edición y tratamiento didáctico: Mujeres matemáticas en México (diseño original nuestro); Las chicas sólo quieren sumar, del programa de TV Los Simpson (editado, con duración de seis minutos y tratamiento didáctico) y Donald en la Tierra de las matemágicas (editado, con tratamiento didáctico).

11 El afecto emerge cuando falla la racionalidad. La ciencia moderna occidental con Immanuel Kant sobrevaloró la racionalidad, categorizando el sentimiento como una expresión infantil y femenina. Por el contrario, consideramos que sólo aquello que nos incumbe afectivamente llega a inquietarnos propiciando motivación o rechazo, pero no indiferencia (González, 2012). 


\section{DISEÑO METODOLÓGICO PARA LA EVALUACIÓN DEL PAMG}

Conjuntamos un enfoque interpretativo del proceso de implementación del PAMG con una evaluación de los resultados (modelo experimental) que permitiera aceptar o rechazar las hipótesis de investigación.

\subsection{DISEÑO CUASIEXPERIMENTAL}

La característica principal de esta modalidad es que los sujetos no son asignados aleatoriamente a las condiciones experimentales; se utiliza en la investigación educativa donde los grupos escolares ya están conformados y es relevante trabajar en ambientes naturales (Jackson, 2008).

\section{Los sujetos}

Se impartió el PAMG a un total de 438 estudiantes de primero de secundaria; con ese fin asistió una facilitadora a cada una de las escuelas, y una investigadora videograbó las seis horas de sesión (dos horas por día) y tomó notas.

El diseño original de la selección de escuelas secundarias de la Ciudad de México fue bietápico (escuela y grupo) al azar. Comprensiblemente, Inmujeres nos solicitó que incluyéramos nueve secundarias con las más bajas calificaciones en la prueba de matemáticas ENLACE; de la selección original conservamos cinco, tres de ellas en colonias clasificadas de baja o muy baja marginalidad y el resto en colonias de muy alta marginalidad. En 12 escuelas se impartió el PAMG y en otra, un programa alterno (educación sexual) como placebo. Ocho escuelas son del turno vespertino y cinco del matutino.

Se intervino en un total de 16 grupos -doce experimentales ${ }^{12}$ y cuatro de comparación- ${ }^{13}$ Se incluyeron dos tipos de grupos de comparación. Tres de ellos son equivalentes; ${ }^{14}$ a éstos no se les impartió el PAMG y sólo se les aplicaron el

\footnotetext{
12 Los grupos en que se implementó el PAMG.

13 Se refiere a los grupos de las mismas escuelas donde sólo se aplicó la escala para medir actitudes hacia las matemáticas y a un grupo en otra secundaria al que se impartió otro programa con similares características pero en otro tema (educación sexual), aplicando la escala antes y después.

14 En educación se considera un grupo equivalente cuando es del mismo grado escolar y de la misma escuela que el grupo experimental; para verificar la equivalencia se calcula la diferencia de medias con la prueba T para muestras independientes.
} 
pre y el postest para controlar el efecto "historia" y el aprendizaje del instrumento de medición y en otro grupo el programa placebo.

\section{El instrumento}

Para evaluar si hubo cambios y en qué sentido, aplicamos la escala Actitudes hacia las matemáticas (EAM) -validada (confiabilidad y validez de contenido) con la población de la Ciudad de México (González, 2005)- a los grupos seleccionados, antes y después de impartir el PAMG.

Para analizar los cambios (pre y post) formulamos varias hipótesis de investigación con un nivel de confianza de 95\% y una potencia estadística de .80 (Cohen, 1992), que más adelante se presentan.

\section{ANÁLISIS DE LA INFORMACIÓN ESTADÍSTICA}

Una vez concluida la etapa de impartición de los programas a los grupos seleccionados, se codificó y capturó la información con ayuda del software estadístico Statistical Package for the Social Sciences (SPSS). Puesto que un aspecto central del análisis era comparar el resultado de la EAM (antes y después del PAMG), tomamos el criterio de suprimir del análisis a aquellos sujetos que no hubieran contestado la EAM en el pre o en el post; ${ }_{i}^{15}$ o también, que no respondieran en tres o más reactivos de la EAM (pre o post), los cuales sumaron 22 casos, quedando un total de 416 estudiantes.

Con el fin de determinar el tipo de prueba estadística que se utilizaría para contrastar medias (pre y post), se analizaron los datos de la EAM (pretest) con la prueba de Kolmogorov-Smirnov para verificar la "bondad de ajuste", y se obtuvieron los siguientes resultados: Z .999, $p=.217$ y $p>.05$; se determinó que la muestra se ajustaba a una distribución normal.

También se analizó con la prueba de Box, la igualdad de las matrices de covarianzas, la cual resultó no significativa ( $F 2.270, p<.000$ ), lo que compromete la confiabilidad de los resultados de la investigación. Ya que esta prueba es muy sensible al tamaño de la muestra, se analizó con otros dos tipos de pruebas

15 Por inasistencia. 
(Traza de Pillai, Lambada de Wiks, Traza de Hotelling), y resultó significativa en ambos casos (1.308, p >.271).

Sobre la base de estos criterios estadísticos, se optó por utilizar la prueba T de Student para grupos relacionados con el fin de analizar las diferencias de medias (pre y post) y la prueba $U$ de Mann-Whitney para mediciones ordinales.

Estimamos también el tamaño del efecto (effect size). Cohen (1992) desarrolló un índice conocido como $d$ que permite conocer no solamente si hay diferencias significativas, sino también la magnitud del efecto. El índice $d$ para las diferencias entre las medias de poblaciones está estandarizado al dividir éste por la desviación típica común.

$$
d=\frac{\bar{Y}_{e}-\bar{Y}_{c}}{s_{c}}
$$

Cohen estableció una serie de rangos, y determinó que en la prueba T un efecto pequeño con d es igual a 0.2 , un efecto mediano es igual a 0.5 y un efecto grande es igual a .8 .

Estos análisis permitieron estimar, a posteriori, la potencia estadística ${ }^{16}$ que habla de la validez de la investigación. Con un efecto de .2 y un nivel de significancia de .05, las tablas de Cohen (1992: 158) señalan que en pruebas de diferencias de media se requiere una población de 393 sujetos para una potencia de .80. La muestra con la que se trabajó fue de 416 sujetos, por lo que se cumple con el criterio señalado por el autor.

\section{IMPLEMENTACIÓN DEL PAMG}

Entre agosto y noviembre del 2011 el PAMG se impartió a estudiantes (47.6\% mujeres) de primer grado de 12 escuelas secundarias. A cada una asistió un facilitador(a) y una observadora, ${ }_{17}^{17}$ trabajando simultáneamente tres escuelas (dos horas por sesión, durante tres días). Las observadoras tomaron nota de cada escuela en un formato diseñado exprofeso y grabaron las sesiones en que se

16 Se refiere a la probabilidad de resultados significativos; es decir, la aceptación de la Hi cuando es verdadera; Cohen (1992) establece como criterio .80 .

17 Cada equipo llevaba consigo laptop, bocinas, videograbadora y tripié. Visitaron previamente cada escuela para entrevistarse con el (la) director(a). 
impartió el PAMG (dos horas por sesión, durante tres días). Las dos facilitadoras se especializan en capacitación laboral (con muchos años de experiencia docente en educación superior, pero sin experiencia con jóvenes de secundaria).

Una vez que se inició la implementación del PAMG, conformamos un laboratorio donde nos reunimos todos los integrantes del equipo para interpretar los videos (70 horas de grabación). En la primera semana hicimos una modificación menor al programa. ${ }^{18}$

\section{PRINCIPALES RESULTADOS}

En el cuadro 1 se presentan los resultados por escuela. En relación con la primera hipótesis de investigación, encontramos diferencias significativas al comparar el promedio de los resultados de la escala (EAM) antes y después, por lo que se acepta la $\mathrm{H}_{1}$ : los estudiantes que trabajan el PAMG incrementaron positivamente sus actitudes hacia las matemáticas, al comparar antes (pre) y después (post) de la intervención (gráfico 1).

Gráfico 1. Promedio de resultados de la EAM antes

y después de impartir el PAMG en grupos experimentales

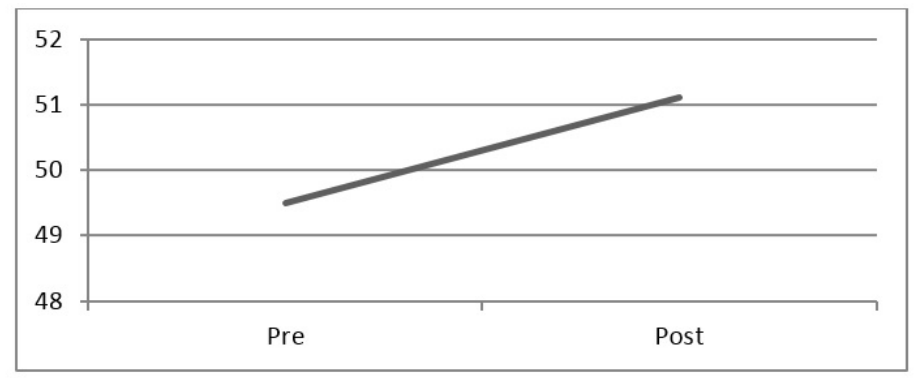

Fuente: Cuadro 1.

En cuanto a la segunda hipótesis de investigación $\left(\mathrm{H}_{2}\right)$, encontramos diferencias significativas positivas en los grupos experimentales (pre y post) con un tamaño

18 En las escuelas con bajo rendimiento en ENLACE, el alumnado no estaba acostumbrado a trabajar en equipo, intercambiando opiniones y poniéndose de acuerdo, por lo que implementamos una actividad para trabajar en pequeños grupos. 
del efecto $d$.20; por el contrario, no encontramos diferencias significativas en los grupos de comparación, con un tamaño del efecto de .08 (cuadro 1), por lo que se acepta la segunda hipótesis de investigación: los estudiantes que trabajan el PAMG incrementaron positivamente sus actitudes hacia las matemáticas, en comparación con aquellos que no lo trabajan o trabajan otro programa (gráfico 2).

Gráfico 2. Promedio de resultados de la EAM antes y después de impartir el PAMG en grupos experimentales y grupos de comparación

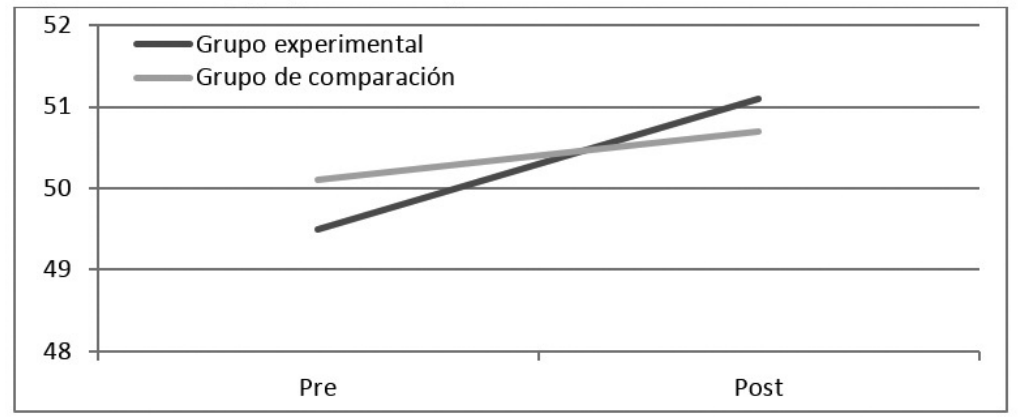

Fuente: Cuadro 1.

\section{DIFERENCIAS POR ESCUELA}

Convencidas de que los resultados generales de las hipótesis de investigación subsumen aspectos particulares de los procesos, identificamos las diferencias por escuela en la EAM, utilizando el índice $d$ para analizar el tamaño del efecto del PAMG en el alumnado. Observamos que en seis escuelas hubo un efecto moderado y en seis fue pequeño o insignificante, valorado con el índice $d$ (cuadro 1). 
Cuadro 1. Media, desviación típica, prueba Ty tamaño del efecto de la Escala de Actitudes hacia las Matemáticas (EAM)

\begin{tabular}{|c|c|c|c|c|c|}
\hline Grupos/escuelas & $\begin{array}{l}\text { Pre media } \\
\text { (DT) }\end{array}$ & $\begin{array}{l}\text { Post media } \\
\text { (DT) }\end{array}$ & $\begin{array}{c}\text { DT } \\
\text { pre y post }\end{array}$ & $\begin{array}{c}\text { T } \\
\text { (nivel de } \\
\text { significación) }\end{array}$ & $\begin{array}{c}\text { Tamaño } \\
\text { del efecto } \\
\text { D (1) }\end{array}$ \\
\hline $\begin{array}{l}\text { Grupo experimental } \\
\mathrm{n}=348\end{array}$ & $\begin{array}{l}49.5 \\
(7.7)\end{array}$ & $\begin{array}{l}51.1 \\
(8.2)\end{array}$ & 8.0 & $\begin{array}{c}-4.931 \\
(* *)\end{array}$ & .22 \\
\hline $\begin{array}{l}\text { Grupo de } \\
\text { comparación } n=91\end{array}$ & $\begin{array}{l}50.1 \\
(7.5) \\
\end{array}$ & $\begin{array}{l}50.7 \\
(7.6) \\
\end{array}$ & 7.7 & $\begin{array}{c}-1.651 \\
\text { (ns) }\end{array}$ & .08 \\
\hline $\begin{array}{l}\text { Grupo experimental } \\
\text { Mujeres } n=165\end{array}$ & $\begin{array}{l}49.7 \\
(7.5) \\
\end{array}$ & $\begin{array}{l}51.1 \\
(8.1) \\
\end{array}$ & 7.9 & $\begin{array}{c}-3.002 \\
(* *)\end{array}$ & .20 \\
\hline $\begin{array}{l}\text { Grupo experimental } \\
\text { Hombres } n=183\end{array}$ & $\begin{array}{l}49.4 \\
(7.9)\end{array}$ & $\begin{array}{l}51.0 \\
(8.3)\end{array}$ & 8.1 & $\begin{array}{c}-2.105 \\
(*)\end{array}$ & .19 \\
\hline 1. Sec. Técnica 45 & $\begin{array}{l}44.8 \\
(4.3)\end{array}$ & $\begin{array}{l}47.0 \\
(5.9)\end{array}$ & 5.3 & $\begin{array}{c}-2.613 \\
\left({ }^{*}\right)\end{array}$ & .41 \\
\hline 2. José Ma. Velasco & $\begin{array}{l}45.3 \\
(8.9) \\
\end{array}$ & $\begin{array}{l}45.9 \\
(7.7) \\
\end{array}$ & 8.1 & $\begin{array}{c}-.512 \\
\text { (ns) } \\
\end{array}$ & .07 \\
\hline 3. León Felipe & $\begin{array}{l}47.2 \\
(6.3) \\
\end{array}$ & $\begin{array}{l}48.8 \\
(7.2) \\
\end{array}$ & 6.8 & $\begin{array}{c}-1.435 \\
\text { (ns) }\end{array}$ & .19 \\
\hline $\begin{array}{l}\text { 4. Sec. Técnica } 115 \\
\text { G. experimental }\end{array}$ & $\begin{array}{l}47.2 \\
(7.4) \\
\end{array}$ & $\begin{array}{l}50.8 \\
(8.6) \\
\end{array}$ & 8.1 & $\begin{array}{c}-3.343 \\
(* *) \\
\end{array}$ & .44 \\
\hline $\begin{array}{l}\text { 4. Sec. Técnica } 115 \\
\text { G. de comparación }\end{array}$ & $\begin{array}{l}47.1 \\
(7.8) \\
\end{array}$ & $\begin{array}{l}48.2 \\
(7.7) \\
\end{array}$ & 7.7 & $\begin{array}{l}-.593 \\
\text { (ns) }\end{array}$ & .14 \\
\hline $\begin{array}{l}\text { 5. Sec. Técnica } 53 \\
\text { G. experimental }\end{array}$ & $\begin{array}{l}53.6 \\
(6.2)\end{array}$ & $\begin{array}{l}55.2 \\
(7.3)\end{array}$ & 6.7 & $\begin{array}{c}-1.501 \\
\text { (ns) }\end{array}$ & .23 \\
\hline $\begin{array}{l}\text { 5. Sec. Técnica } 53 \\
\text { G. de comparación }\end{array}$ & $\begin{array}{l}48.6 \\
(9.0) \\
\end{array}$ & $\begin{array}{l}50.3 \\
(7.2) \\
\end{array}$ & 8.1 & $\begin{array}{c}-1.426 \\
\text { (ns) }\end{array}$ & .20 \\
\hline $\begin{array}{l}\text { 6. Sec. Técnica } 70 \\
\text { G. experimental }\end{array}$ & $\begin{array}{l}50.6 \\
(6.8) \\
\end{array}$ & $\begin{array}{l}53.7 \\
(7.7) \\
\end{array}$ & 7.3 & $\begin{array}{c}-2.498 \\
\left(^{*}\right)\end{array}$ & .42 \\
\hline $\begin{array}{l}\text { 6. Sec.Técnica } 70 \\
\text { G. de comparación }\end{array}$ & $\begin{array}{l}49.7 \\
(6.9) \\
\end{array}$ & $\begin{array}{l}50.0 \\
(7.4) \\
\end{array}$ & 7.0 & $\begin{array}{c}-.350 \\
\text { (ns) } \\
\end{array}$ & .04 \\
\hline 7. Rep. de Cuba & $\begin{array}{l}50.2 \\
(7.2) \\
\end{array}$ & $\begin{array}{l}51.2 \\
(7.2) \\
\end{array}$ & 7.2 & $\begin{array}{c}-.511 \\
\text { (ns) }\end{array}$ & .13 \\
\hline 8. Carlos A. Carrillo & $\begin{array}{l}50.6 \\
(7.1) \\
\end{array}$ & $\begin{array}{l}51.4 \\
(6.6) \\
\end{array}$ & 6.8 & $\begin{array}{c}-.494 \\
\text { (ns) }\end{array}$ & .11 \\
\hline
\end{tabular}




\begin{tabular}{l|c|c|c|c|c}
\hline 9. Sec. Técnica 60 & $\begin{array}{c}51.2 \\
(7.1)\end{array}$ & $\begin{array}{c}51.4 \\
(9.5)\end{array}$ & 8.4 & $\begin{array}{c}-.191 \\
(\mathrm{~ns})\end{array}$ & .02 \\
\hline 10. Sec.Técnica 31 & $\begin{array}{l}52.9 \\
(6.9)\end{array}$ & $\begin{array}{c}55.2 \\
(6.4)\end{array}$ & 6.7 & $\begin{array}{c}-2.803 \\
(* *)\end{array}$ & .34 \\
\hline 11. Dr. Manuel Barranco & $\begin{array}{l}50.7 \\
(8.4)\end{array}$ & $\begin{array}{c}52.8 \\
(9.3)\end{array}$ & 8.8 & $\begin{array}{c}-2.377 \\
(*)\end{array}$ & .23 \\
\hline 12. Martín V. González & $\begin{array}{l}51.4 \\
(8.2)\end{array}$ & $\begin{array}{c}51.4 \\
(8.4)\end{array}$ & 8.2 & $\begin{array}{c}-.043 \\
(\mathrm{~ns})\end{array}$ & .03 \\
\hline 13. Diego Rivera & 52.5 & 52.9 & 7.0 & $\begin{array}{c}-.509 \\
(\mathrm{~ns})\end{array}$ & .05 \\
\hline
\end{tabular}

El nivel de significación es un estadístico de prueba en virtud del cual se concluye acerca de la existencia del fenómeno estudiado, o el riesgo de rechazar erróneamente la Ho.

$\left(^{* *}\right)$ Significativo al 1\%; $\left(^{*}\right)$ Significativo al 5\%; (ns) No significativo.

Hasta .20, efecto pequeño; entre .21 y .50, efecto moderado; 51 o más, efecto grande (Cohen, 1992).

Constatamos que en las escuelas en donde se impartieron menos de cuatro horas del PAMG, ${ }^{19}$ con diversos inconvenientes como la entrada y salida de personas, interrupciones (simulacro sismo, examen, etc.) (núms. 9, 12 y 13), el efecto fue insignificante.

Encontramos diferencias importantes $(T-4.236, \mathrm{p}<.001)$ al comparar el factor $d$ por turnos, a favor de escuelas del turno vespertino. Este hecho se ha mencionado en otras investigaciones: quienes reciben menos educación ${ }^{20}$ tienden a responder más positivamente (Forgasz, 2010).

Particularmente nos preguntamos cómo fue que las estrategias del PAMG mejoraron de manera significativa la actitud hacia las matemáticas en la mitad de las escuelas y por qué en otras seis no hubo cambios estadísticamente significativos. Retomamos las grabaciones (70 horas), y procedimos de la siguiente forma:

a) Observamos en dos ocasiones cuatro videos de las tres sesiones (uno por cada facilitador), tomando nota de lo más significativo de cada actividad (contexto, situación, formas de comunicación entre el (la) facilitador(a) y

19 En un caso por simulacro de sismo, en otro porque el director argumentó no haber recibido el oficio y en uno más por pérdida de las llaves del salón de cómputo, no se impartieron las seis horas programadas.

20 Las secundarias vespertinas -en comparación con las matutinas- tienen menos alumnas, son grupos más reducidos y hay estudiantes mayores de 13 años. Por no tratarse de una muestra aleatoria, no analizamos diferencias por turno. 
el grupo y el alumnado, respuesta del alumnado ante cada actividad didáctica propuesta). ${ }^{21}$

b) Formulamos algunas conjeturas acerca de las escenas, que fuimos aclarando y enriqueciendo con la observación de otros videos. Comentamos estas inferencias con las investigadoras y las facilitadoras participantes para conocer su punto de vista.

c) Continuamos interpretando videos de otras escuelas, hasta que decidimos que era suficiente lo que muestran las escenas, para validar las conjeturas.

d) Por último, contrastamos algunos aspectos de las escuelas donde mejoraron las actitudes del alumnado hacia las matemáticas (facilitador, formas de relación, infraestructura, etc.) con las escuelas donde no hubo avances importantes.

\section{B) INTERPRETACIÓN DEL PROCESO DE IMPLEMENTACIÓN DEL PAMG}

En el laboratorio revisamos las grabaciones en las que iniciamos con dos preguntas generales: ¿cómo se llevó a cabo la actividad? y ¿qué efecto producían las diferentes estrategias didácticas programadas? Si bien en la mayoría hubo una buena recepción, en dos escuelas el video dura poco por "no haber recibido el oficio a tiempo".22

A algunas de las escuelas vespertinas se les conoce como "escuelas basurero", que es revelador de la mala opinión que se tiene tanto de la escuela como del alumnado que ahí llega. En todas las escuelas se contaba con un espacio para la proyección de videos, y se identifican tres tipos: a) salón tipo teatro con butacas fijas; b) sala de cómputo con mesas; c) espacio en la biblioteca, con mesas movibles. La mejor opción para trabajar el PAMG fue la c y la peor la a.

La actitud del alumnado fue en algunos casos de confrontación (malas palabras, parejas besándose, riéndose y haciendo tanto ruido que era imposible escucharse); después de un rato, entre ellos empezaban a callarse y pedir atención.

21 En esta primera observación no contábamos con información acerca de en qué grupos habían mejorado las actitudes y en cuáles no.

22 Las investigadoras asistieron una semana antes de la actividad a cada escuela para presentarse con el directivo y conocer las instalaciones en que se iba a implementar el PAMG. 


\section{ESTRATEGIAS DIDÁCTICAS PARA EL CAMBIO DE ACTITUDES HACIA LAS MATEMÁTICAS ${ }^{23}$}

Cada actividad se correspondía con un tipo de estrategia didáctica (gráfico 3); a continuación se describe cada una de las actividades.

\section{PERSUASIÓN}

Los videos lograron captar el interés y emocionaron a buena parte del alumnado. Dieron excelentes resultados para problematizar algunas creencias en relación con las matemáticas. El video Donald, a pesar de haberse producido hace más de 80 años (1935), continúa siendo vigente para mostrar una dimensión cultural de hacer matemáticas; particularmente persuade en el sentido de que las matemáticas son útiles para la vida cotidiana y que no se trata solamente de un saber abstracto sin relación con el mundo: la música, la arquitectura o el juego de estrategia (ajedrez).

Por su parte, el video Mujeres matemáticas en México atrajo la atención del grupo y permitió dar a conocer las aportaciones que algunas mujeres han hecho al campo de las matemáticas. Debido al alto volumen de la música, no se escucha con claridad el mensaje de la voz; opinaron que es un video corto y claro para los fines que persigue, según los comentarios de las alumnas al concluir: "Ahí faltamos nosotras".

Una situación muy desagradable que se repitió en todos los grupos fue la burla racista que genera la imagen de la primera matemática afroamericana Evelyn Boyd ("qué fea"; "ja ja, se parece a tu abuela"). El tema de la discriminación racial en México es un problema muy presente y denunciado, aunque poco trabajado en la escuela, e implicaría por sí mismo destinarle no sólo algunas horas en la clase, sino una práctica cotidiana involucrando a todos los agentes educativos.

El video Trabajo en equipo, el cual se incluyó debido a que la mayoría de los grupos vespertinos no están acostumbrados a esta forma de actividad, les fue significativo cuando evaluaron el PAMG: "Aprendí que es importante trabajar en equipo".

23 El PAMG se puede consultar en González (2013). 


\section{CONFLICTO COGNITIVO}

El video de dibujos animados de la Familia Simpson Las niñas solo quieren sumar relata que a Juliana, quien fue alumna de la escuela primaria de la ciudad de Springfield, le organizan una ceremonia en el auditorio para reconocer sus éxitos profesionales. El director Skinner recuerda en su exposición que ella fue muy buena alumna; Juliana interrumpe y comenta: "Bueno, no tanto en matemáticas", a lo que Skinner responde: "Bueno, es lógico, eres mujer... lo que quise decir es que los niños son mejores en Matemáticas, Ciencias, materias de verdad". Posteriormente presentan cómo separan a niños y niñas en la clase de Matemáticas. Lisa se disfraza de niño para entrar a las clases que a ellos les imparten, ya que en la de niñas sólo se preocupan por su autoestima y no les enseñan a resolver problemas.

La reacción de las alumnas no se hace esperar, quienes se apresuran a señalar que ellas son mejores en las clases de Matemáticas. ${ }^{24}$ El mensaje de este video consigue que hablen en primera persona expresando su disgusto. En el caso de los alumnos, no se dan por aludidos, aunque muestran atención al tema.

La sola exposición de los videos no produce el efecto esperado; es importante el trabajo didáctico, en el que se les formulan preguntas específicas que apuntan a la reflexión de sus propios pensamientos. ${ }^{25}$

\section{METACOGNICIÓN}

La reflexión ${ }^{26}$ y el diálogo son centrales en esta estrategia; si el alumnado reflexiona acerca del método que sigue para resolver un problema y este proceder le resulta clarificador, es posible que modifique sus creencias acerca de las matemáticas (son difíciles) y que le genere placer afrontar el reto.

Por su parte, el diálogo, a decir de Hans-Georg Gadamer, entra en el marco de la pregunta-respuesta, de la llamada y la escucha, elementos entrañablemente

24 Esto es cierto, ya que el alumnado de secundaria en las escuelas es evaluado por sus profesores (as), quienes en promedio califican mejor a las alumnas que a los alumnos (González, 2005).

25 Por ejemplo, en el video de Mujeres matemáticas en México, a la pregunta de la facilitadora si conocían alguna mujer matemática comenta un estudiante "yo pensé que no había matemáticas famosas", a lo que algunos asienten. Nuevamente les interroga, ¿por qué pensaban eso? "no conocía a ninguna"; y por qué será que no conocías a ninguna "bueno, tampoco conocía a matemáticos, ja ja."

26 Entendida como "una continua interacción entre el pensamiento y la acción” (Schön, 1983). 
unidos. Las posibilidades de dialogar entre facilitador (F) y estudiantes (E) y de E entre ellos (as) pasa porque se interroguen y se escuchen, y esto sólo es posible en pequeños grupos.

La actividad "Número secreto" pretendía mostrar que resolver problemas puede implicar complejidad, pero también diversión, cuando se consigue. Si alguna actividad se trabajó en equipo -entendiendo por esto no sólo un pequeño número de estudiantes reunidos, sino la participación colectiva en que cada cual realizó una parte para conseguir resolver el problema que se les planteaba-, fue "número secreto" que incluyó diálogo y reflexión. Resultó muy gratificante ver cómo varios equipos se mostraban satisfechos de sus resultados.

"Encriptación de mensajes"27 pretendió vincular el cifrado y descifrado de mensajes con la resolución de problemas que implica análisis, reflexión y descubrimiento. Resultó interesante y divertida para buena parte del alumnado, que se sorprendió por la creación de códigos creativos y mensajes positivos que manifiestan afecto ("quiero decirles que los quiero"). Esta actividad fue la que menos comprendieron el facilitador y las facilitadoras y en la cual mostraron su desconocimiento de la enseñanza de las matemáticas en el momento del cierre.

El conocimiento que el facilitador tenía del programa incidió positivamente: los dos que participaron en el diseño del PAMG tuvieron mejores resultados. Las formas de relación y comunicación entre el alumnado (conocido como clima escolar) fueron otro aspecto que favoreció mejores resultados.

\section{MÉTODO DE CASO}

"La mejor decisión" consiste en que por equipos el alumnado elija, de tres compañías de telefonía celular cuyas tarifas de prepago se le proporcionan, cuál de ellas es la más conveniente y por qué. Dicha estrategia ofrece la posibilidad de vincular el contenido temático con el entorno social y aplicar los conocimientos a situaciones cotidianas.

La actividad no resultó muy interesante ni divertida, pues al estar en equipos se comunican poco y no saben ponerse de acuerdo. Además, dos de las facilitadoras no comprendieron bien el sentido de la actividad: ${ }^{28}$ mostrar que, si se clarifican diferentes aspectos de la oferta de cada empresa telefónica (costo,

27 Actividad sugerida por la doctora Gisela Espinosa.

28 Una se enfocó más en estimar el costo por llamada y la otra no propiciaba la reflexión. 
atractivo del celular, calidad de comunicación, etc.), se pueden tomar decisiones informadas.

"Cuadro cubista" fue la actividad que más interesó al alumnado, ya que es una de las que apuntan precisamente a la dimensión afectiva. Se les pide que dibujen una pintura en la que ocupen figuras geométricas. Se les explica brevemente quién y dónde se creó lo que se conoce como dibujo cubista. La finalidad era reforzar la idea de que las matemáticas no son solamente algoritmos o conocimiento sin sentido para la vida cotidiana.

El dibujo logró que el alumnado expresara muy diferentes imágenes y sentimientos y diera rienda suelta a la creatividad: frecuentemente dibujaron grafitis ${ }^{29}$ (en otros casos muestran dibujos simples o convencionales, como casas o personas). A algunos equipos incluso hubo que pedirles varias veces que terminaran el trabajo, pues el tiempo destinado a otras actividades había concluido.

Consideramos que se ha menospreciado la comunicación gráfica como medio de expresión y comunicación en este nivel educativo.

\section{HISTORIA DE VIDA}

Originalmente se planteó que en pequeños grupos cada estudiante hablara de su experiencia personal con las matemáticas; pronto constatamos que no están acostumbrados a hablar en primera persona (burlas, desinterés de compañeros), por lo que hicimos algunas modificaciones y pedimos que tres hablaran de experiencias positivas y tres de negativas, intentando propiciar la reflexión al respecto. Las experiencias negativas fueron especialmente conmovedoras.

Relacionamos cada uno de los reactivos (pre y post) de los resultados en la Escala con la prueba no paramétrica de Wilcoxon; a continuación presentamos las actividades y estrategias didácticas, el objetivo y los reactivos, reportando solamente aquellos con un nivel de significación de 5\% o menor.

29 Los grafitis suelen asociarse a la violencia juvenil, parece que es una de las formas de comunicación de estos jóvenes. 
Cuadro 2. Nivel de significancia estadística en la relación de actividad, objetivos y reactivos

\begin{tabular}{l|l|l|c}
\hline $\begin{array}{l}\text { Actividades PAMG y } \\
\text { estrategias didácticas }\end{array}$ & Objetivo & Reactivos & $\begin{array}{c}\text { Nivel de } \\
\text { significancia }\end{array}$ \\
\hline $\begin{array}{l}\text {-Video familia Simson } \\
\text {-Video mujeres } \\
\text { matemáticas(metacognición y y } \\
\text { persuasión) }\end{array}$ & $\begin{array}{l}\text { Incrementar la auto- } \\
\text { confianza en sus } \\
\text { capacidades } \\
\text { matemáticas }\end{array}$ & $\begin{array}{l}\text { 8. Mi habilidad para } \\
\text { las matemáticas es } \\
\text { muy buena. } \\
18 \text { Soy bueno (a) en } \\
\text { matemáticas. }\end{array}$ & $* *$ \\
\hline $\begin{array}{l}\text {-Número secreto } \\
\text {-Encriptación de mensajes } \\
\text { (metacognición) }\end{array}$ & $\begin{array}{l}\text { Cuestionar la idea de } \\
\text { que las matemáticas } \\
\text { son difíciles }\end{array}$ & $\begin{array}{l}\text { 14. Me parecen muy } \\
\text { fáciles las } \\
\text { matemáticas. }\end{array}$ & $*{ }^{* *+}$ \\
\hline $\begin{array}{l}\text {-Toma de decisiones (método } \\
\text { de caso) }\end{array}$ & $\begin{array}{l}\text { Presentar las } \\
\text { matemáticas como un } \\
\text {-Donald y la Tierra de las } \\
\text { Matemágicas (persuasión) }\end{array}$ & $\begin{array}{l}\text { 1. Las matemáticas me } \\
\text { ayudan a tomar } \\
\text { decisiones }\end{array}$ & $*{ }^{* *}+$ \\
\hline $\begin{array}{l}\text {-Dibujo cubista (trabajo en } \\
\text { equipo) }\end{array}$ & $\begin{array}{l}\text { Disminuir el temor } \\
\text { que genera hacer } \\
\text { matemáticas }\end{array}$ & $\begin{array}{l}\text { 11. Me siento } \\
\text { intranquilo (a) en clase } \\
\text { de matemáticas. }\end{array}$ & $*$ \\
\hline $\begin{array}{l}\text {-Dibujo cubista (trabajo en } \\
\text { equipo) } \\
\text {-Gusto por las matemáticas } \\
\text { (historia de vida) }\end{array}$ & $\begin{array}{l}\text { Considerar interesante } \\
\text { y divertido la } \\
\text { resolución de } \\
\text { problemas }\end{array}$ & $\begin{array}{l}\text { 2. Las matemáticas } \\
\text { pueden ser divertidas. }\end{array}$ & $*$ \\
\hline
\end{tabular}

$\left(^{* *}\right)$ Significativo al 1\%; $\left({ }^{*}\right)$ Significativo al 5\%

(+) Especialmente para las alumnas; (++) especialmente para los alumnos

Por último, correlacionamos algunas variables como cantidad de estudiantes por grupo, clima del aula ${ }^{30}$, índice de feminidad y prueba Enlace con el puntaje D y promedio de resultados en EAM (pre) identificando relación entre puntaje D y clima de aula y (cuadro 3).

30 Esta variable surgió en la observación de los videos en el laboratorio; nos percatamos que un ambiente socio-afectivo positivo de interacción en el aula favorecía la implementación del PAMG en el grupo; posteriormente, le asignamos un valor a cada grupo ( $1=$ muy malo y $5=$ muy bueno). 
Cuadro 3. Correlación entre variables seleccionadas

\begin{tabular}{|c|c|c|c|c|c|c|}
\hline Variables & 1 & 2 & 3 & 4 & 5 & 6 \\
\hline 1.Puntaje D & & -193 & -148 & $.782^{* *}$ & -.388 & -.228 \\
\hline 2.EAM (Pre) & & & .106 & .191 & .478 & $.721^{* *}$ \\
\hline 3. No. estudiantes por gpo. & & & & .152 & .002 & -.007 \\
\hline 4.Clima del aula & & & & & -103 & .147 \\
\hline 5.Indice de feminidad & & & & & & .204 \\
\hline 6. Prueba ENLACE & & & & & & \\
\hline
\end{tabular}

$\left({ }^{* *}\right)$ Significativo al $1 \% ;\left(^{*}\right)$ Significativo al 5\%

\section{APRENDIZAJES DEL PAMG}

Enseguida se describen algunos aprendizajes que nos dejó la implementación del PAMG, mediante la interpretación de los videos:

1. Las actividades funcionan mejor con instrucciones claras y cortas; cuando se dificulta la comprensión es bueno poner un ejemplo con un grupo para que todos observen.

2. El mejor antídoto para la indisciplina son las actividades que le hacen sentido y le resultan interesantes al alumnado, cuando menos a la mayoría.

3. Muy diversos factores influyeron en mejorar las actitudes del PAMG: la compresión del programa; estilo de comunicación y enseñanza del facilitador; grupos con aceptable relación entre ellos(as) y no mayormente apáticos con el aprendizaje (buen ambiente de aula); contar con un aula con mobiliario movible para trabajar en pequeños grupos, libre de interferencias, y haber cubierto el total de actividades didácticas señaladas en el PAMG

4. No cubrir todas las actividades del PAMG, malas instalaciones y mal ambiente de aula afectaron el cumplimiento de los objetivos en seis escuelas.

5. La posibilidad de que el alumnado sea el protagonista en la formación pasa por el trabajo en equipo previamente planificado.

Al finalizar la impartición del PAMG se pedía al alumnado que evaluara los tres días de trabajo, preguntándoles qué aprendieron y cómo se sintieron. Tres aspectos 
destacan en sus respuestas en lo general: a) fue divertido hacer matemáticas; b) las mujeres pueden ser tan buenas como los hombres en matemáticas; c) las ventajas del trabajo en equipo. A continuación, algunos ejemplos.

Ana Angélica: "Que las mujeres también pueden estudiar matemáticas".

María Fernanda: "A trabajar en equipo y que las mujeres son igual de buenas para las matemáticas que los hombres..., me sentí bien, tranquila".

Raymundo: "Aprendí a convivir en equipo y mucho de matemáticas... me sentí alivianado y bien".

Cercle: "Se me hizo muy interesante lo del pictograma... gracias por venir a enseñarnos, a hacernos entender que podemos convivir en equipo y la importancia de las matemáticas".

Giovanny: "Con muchas ganas de aprender más sobre las matemáticas".

\section{DISCUSIÓN}

Al finalizar la evaluación (experimental) confirmamos que cumplimos con el objetivo de la intervención que pretendía mejorar significativamente las actitudes hacia las matemáticas en alumnas de secundaria a través del PAMG, programa que diseñamos a partir del análisis conceptual de actitud, del cual derivamos estrategias didácticas que incluyeron las dimensiones creencias cognitivo-afectivas aunadas y relacional, obteniendo mejoras en el sentido de resignificar a las matemáticas (reactivos 1 y 2: Las matemáticas pueden ser divertidas, Me ayudan a tomar decisiones) y resignificar-se en relación con sus habilidades en la disciplina (reactivo 8: Mi habilidad para las matemáticas es muy buena) y disminuir la ansiedad que les genera (reactivo 11: Me siento intranquila en clase de matemáticas).

Al analizar por escuelas, en seis de las doce escuelas encontramos que mejoraron significativamente sus actitudes hacia las matemática con 99\% de confianza y en 3 escuelas más el efecto fue pequeño.

El conceptualizar cognición-afecto como dos caras de una misma moneda y la dimensión personal-relacional humana iluminaron aspectos que 
usualmente las categorías individuo, cognición y emoción no permiten observar ni trabajar pedagógicamente.

Al incorporar una perspectiva de género a través de dos videos -la participación histórica de mujeres en el campo de las matemáticas y una caricatura en donde el director de una escuela descalifica a una exalumna señalando que era buena estudiante "pero no en materias de verdad, como matemáticas"- generó una reacción de rechazo en buena parte de las alumnas quienes mostraron abiertamente su negativa a tal afirmación, actividades que seguramente incidieron en las respuestas en la post aplicación del cuestionario en torno a que es un conocimiento propio para varones y que las mujeres han ingresado a este campo recientemente.

Las escuelas vespertinas -con más bajos puntajes en matemáticas previo a la intervención- fueron las que en mayor medida mejoraron sus actitudes hacia las matemáticas y el estereotipo de que es un saber sin relación con la vida cotidiana, confirmando lo señalado por otras investigaciones en el sentido de lo provechoso de destinar más y mejores recursos a quienes cuentan con menos oportunidades.

La actividad que incluimos para trabajar en equipo -con un video que no dura más de tres minutos-, aunado a la forma en que se movieron las bancas para conformar los equipos en aquellos salones que lo permitían fueron favorables para la comunicación y trabajo en pequeños grupos; así mismo, en algunas ocasiones se logró una aceptable-buena comunicación a través de los dibujos que sería deseable investigar en futuras investigaciones con jóvenes.

Fue un error subestimar el saber del profesorado de secundaria el cual lleva años; a pesar de que facilitadores llevan muchos años de experiencia formando profesionistas fueron evidentes sus limitaciones en las dos primeras semanas para trabajar con jóvenes de secundaria; también la formación pedagógica de uno de ellos y su involucramiento con el diseño del programa.

Dos de las actividades -número secreto e encriptación de mensajes- se relacionaron positivamente con la idea de que las matemáticas son difíciles, especialmente para los alumnos. El video de Donald favoreció que el alumnado contemplara las matemáticas con una actividad útil y valiosa.

Consideramos que la intervención resultó novedosa en más de un sentido. Desde una perspectiva didáctica, la formación de actitudes ha tomado realce en la última década, pero aún hay escasa investigación acerca de cómo transformar positivamente las actitudes hacia el aprendizaje, sobre todo en el caso particular de las matemáticas; al respecto, probamos dos estrategias didácticas innovadoras (persuasión y cuéntame tú tu historia) que mostraron ser efectivas, la primera en 
el análisis experimental y la segunda en el interpretativo en donde el alumnado se expresa con mayor amplitud la valoración que hace del curso que recibió.

Desde un enfoque ético-político, el PAMG pretende garantizar la igualdad de derechos y oportunidades para niñas y mujeres en este campo de conocimiento; logramos que las alumnas mejoraran sus actitudes hacia las matemáticas conociendo a mujeres sobresalientes en esta materia, lo que les permitió cuestionarla como una actividad propia de los varones.

Metodológicamente, se probaron maneras de investigar integrando perspectivas antagónicas -un diseño cuasiexperimental y un análisis interpretativo- y haciéndolas coincidir en una propuesta para mejorar la comprensión/explicación de fenómenos complejos como la intervención educativa.

Hubiera sido deseable aplicar de nuevo el cuestionario de actitudes al alumnado dos años después, ya que ignoramos el efecto del PAMG a más largo plazo; sin embargo, en México los estudios de este tipo en educación prácticamente son inexistentes.

\section{AGRADECIMIENTOS}

Esta investigación fue solicitada y financiada en parte por el Instituto Nacional de las Mujeres de México.

\section{REFERENCIAS}

Arthur, J., Waring, M. Coe R. y Hedges I. (2012). Research Methods and Methodologies in Education. Thousand Oaks, CA: Sage Publications.

Bolívar, A. (2000). El desarrollo de actitudes. En Coll, C. El constructivismo en la práctica. Grao. Barcelona, 47-55.

Bolívar, A. (2005). El conocimiento de la enseñanza: explicar, comprender y transformar. Revista Mimesis-Ciências Humanas (Bauru-São Paulo). Revista de currículum y formación del profesorado, 25 (1),17-42. Consultado el 8 de junio de 2010 en: http:// www.edusc.com.br/colecoes/revistas/mimesis/index.htm

García, R. M. y Sebastián Ch. (2011). Creencias epistemológicas de estudiantes de Pedagogía en educación parvularia, básica y media: ¿diferencias en la formación inicial docente? Psykhe, 20 (1), 29-43. 
Gil, N., Blanco, L. y Guerrero, E. (2005) El dominio afectivo en el aprendizaje de las matemáticas. Una revisión de sus descriptores básicos. UNION Revista Iberoamericana de Educación Matemática (2) 15-32

Cohen, J. (1992). A Power Primer. Psychological Bulletin, 112, 155-159.

Chiozza, L. (1998). Cuerpo, afecto y lenguaje. Alianza. Buenos Aires.

Meece, J. L., Eccles-Parsons, J., Kaczala, C. M., Goff, S. B., y Futterman, R. (1982). Sex differences in math achievement: Toward a model of academic choice. Psychological Bulletin, 91, 324-348. Meehan, A. M. (1984).

Eccles-Parson, J., Kaczala, S. B. Goffy R. Futterman (1982). Sex Differences in Math Achievement: Toward a Model of Academic Choice. Psychological Bulletin 92, 324-348.

Espinosa, C. (2010). Diferencias entre hombres y mujeres en educación matemática: ¿qué pasa en México? Investigación y Ciencia, núm. 46, pp. 28-35.

Forgasz, H. J. (2010). International Perspectives o non Gender and Mathematics Education. Information Age Publishing. Charlotte, NC.

Gómez-Chacón, I. M. (2000). Matemática emocional: los afectos en el aprendizaje matemático. Narcea. Madrid.

González, R. M. (2004a). Género y educación: resignificando una historia. Formación del profesorado de educación básica. Decisio Revista del Crefal 2, 25-32.

González, R. M. (2004b) Género y matemáticas: balanceando la ecuación. Porrúa/Universidad Pedagógica Nacional. México.

González, R. M. (2005). Un modelo explicativo del interés hacia las matemáticas de las y los estudiantes de secundaria". Revista Educación Matemática 17, 107-128.

González, R. M. (2006). Mujeres matemáticas: análisis del caso de México. Cuestiones de Género: de la lgualdad a la Diferencia 1, 113-136.

González, R. M. (2012). Paradojas de trabajar género en educación: algunas reflexiones acerca de la formación o cómo salir del gatopardo. En Jorge Luis Silva (coordinador). Género y educación: aportes para la discusión jurídica. Suprema Corte de Justicia de la Nación/ Fontamara. México.

Organización para la Cooperación y el Desarrollo Económicos, OCDE (2016). Panorama de creencias, expectativas y motivación de los estudiantes. Informe de resultados de PISA 2015. Consultado el 30 de noviembre de 2016 en: https://www.oecd.org/pisa/ PISA-2015-Mexico-ESP.pdf

Planes y programas de estudio para educación secundaria (2006). Diario Oficial de la Federación, 26 de mayo. Consultado el 30 de abril de 2011 en: https://www.sep.gob. mx/work/models/sep1/Resource/ca8cef5b-610b-4d55-8a52-03f1b84d0d6c/a384.pdf Jackson, L. S. (2008). Research Methods and Statistics: A Critical Thinking Approach. Wadsworth Cengage Learning. Belmont, CA. 
Leder, G. C. y Forgasz, H. J. (2002). Measuring Mathematical Beliefs and their Impact on the Learning of Mathematics: A New Approach. En Gilah C. L., E. Pehkonen y G. Töner (editores). Beliefs: A Hidden Variable in Mathematics Education? Kluwer Academic Publishers. Dordrecht, The Nertherlands.

McLeod, D. (1992). Research on Affect in Mathematics Education: A Reconceptualization. En Handbook of Research on Mathematics Teaching and Learning. McMillan Library. Nueva York.

McLeod, D. y McLeod S. (2002). Synthesis-Beliefs and Mathematics Education: Implications for Learning, Teaching and Research. En Gilah C. L., E. Pehkonen y G. Törner (Eds). Beliefs: A Hidden Variable in Mathematics Education? Kluwer Academic Publishers. Dordrecht, The Nertherlands.

McGraw, R., S. Lubienski y M. Strutchens (2006). A Closer Look at Gender in naep Mathematics Achievement and Affect Data: Intersections with Achievement, Race/Ethnicity, and Socioeconomic Status. Journal for Research in Mathematics Education 37, 129-150.

Menchaca, A. (2000). Las ciencias exactas en México. Fondo de Cultura Económica. México.

Murillo, F. J. (2006). Un marco comprensivo de mejora de la eficacia escolar. Revista Mexicana de Investigación Educativa 21, 319-359.

Murillo, F. J., R. Hernández-Castilla y C. Martínez-Garrido (2016). ¿Qué ocurre en las aulas donde los niños y niñas no aprenden? Estudio cualitativo de aulas ineficaces en Iberoamérica. Perfiles Educativos 151, 55-70.

Ricoeur, Paul (2011). Sí mismo como otro. Siglo XXI Editores. México.

Shön, D. (1983). The Reflective Practitioner: How Professionals Think in Action. Temple Smith. Londres.

Ursini, S. Ramírez, M. Rodríguez, C. Tigueros, M. y Lozano, M. (2010). Studies in Mexico on Gender and Mathematics. En Forgasz, H. (Ed) International Perspectives on Gender and Mathematics Education. Charlotte, NC: Information Age Pub.

Ursini, S., Montes, D. Ramírez, M. y García, S. (2012). Actitudes hacia el estudio de las matemáticas. En Carvajal E. (Ed). Matemáticas para profesores de Preescolar y Primaria. UNAM/Editorial Siglo XXI. México.

ROSA MARÍA GONZÁLEZ JIMÉNEZ

Dirección: Pirineos 242 Depto. 205 Col. Santa Cruz Atoyac,

Delegación Benito Juárez CP 03310

Teléfono: $\quad 5544133$ 